\title{
A new approach of online waste removal process for laminated object manufacturing (LOM)
}

\author{
Y.S. Liao*, L.C. Chiu, Y.Y. Chiu \\ Department of Mechanical Engineering, National Taiwan University, Taipei, Taiwan
}

\begin{abstract}
A new online de-cubing laminated object manufacturing process which employs self-adhesive sheet with shielding paper is proposed in this paper. The main idea of this new process is to perform bond-then-cut at the same position, avoiding the accumulated error resulted from reposition, and to de-cube the inner waste material in process by peeling off the shielding paper. By doing so, about $30-80 \%$ waste material according to various geometric shapes can be removed during the machining process. The new process not only shortens the time for laser cutting and de-cubing, but also enables the production of hollow and shell-shaped parts. Hence, the proposed process promotes both the performance efficiency of laminated process and the manufacturing ability regardless the geometry of the parts.
\end{abstract}

(C) 2003 Elsevier B.V. All rights reserved.

Keywords: Rapid prototyping; LOM; Layer manufacturing; Online de-cubing

\section{Introduction}

The laminated object manufacturing (LOM) is one of the rapid prototyping (RP) technologies. It has the features of cheap material, fast manufacturing and simple process. The LOM technology adopts layer manufacturing process, using mainly papers with thermoplastic adhesive as laminating material. During machining process, the adhesive is faced downward and a hot roller is used on a new layer of paper to heat up the adhesive, bonding the two layers of paper tightly.

However, paper-laminating LOM technology has many shortcomings and difficulties in manufacturing process. The LOM process performs bond-then-cut, employing waste material as its support, making waste material coexistent with the workpiece and needed to be removed after the machining process is finished. It is not only skilled labor dependent and time consuming, but also damages the workpiece easily. Besides, many geometry shapes (such as hollow and vase parts) are hardly produced by LOM process because of the inner waste material. It is also a consumption of laser power to perform crosshatching and de-cubing.

Cho and Lee [1] proposed a new sheet deposition type rapid prototyping system. The material is cut twice in different positions during one pass for de-cubing, and steps are followed repeatedly until the desired part is obtained. The

\footnotetext{
* Corresponding author. Fax: +886-2-2366-0443.

E-mail address: liaoys@ntu.edu.tw (Y.S. Liao).
}

supporting algorithm brought up by Hur and Lee [2] is employed to support and position the workpiece after the material is cut. Although the process is effective for de-cubing, there arises another two problems: (i) cut-then-bond process will cause a loss in positioning precision and the accumulated error becomes more serious as the layers are increased; (ii) there is still much waste material left in the direction of $z$-axis. In addition, this algorithm cannot be used in many geometry shapes.

Chiu et al. [3] proposed a new process, positioning the material on an $X Y$-plane to perform cut-then-bond, and a rule of bridge-building to support the workpiece after waste material is de-cubed. In this way, online de-cubing can be achieved without accumulated error; even so, the positioning error in $z$-axis is still existent, and deflections resulted from the weight of inner contour is left undiscussed.

Concerning the raw material used in LOM, Klosterman et al. [4] made a study of ceramics and compound material; Chi et al. [5] developed a metal LOM technology; Lone Peak Engineering Inc. [6] produced a metal tape for LOM process. All these studies strengthened the workpiece but made it more difficult to de-cube the waste material.

In view of the restrictions of geometry shapes that can be made, and of the important role played by waste material de-cubing in LOM process, this study aims at better manufacturing ability of geometric pieces and the improvement of waste material de-cubing. An online de-cubing process, which can de-cube the inner waste material online, is proposed in this study. A rule of support, dubbed 
as "bridge-supporting algorithm", which builds bridges to support and position the workpiece after the material is cut, is developed as well. The proposed process performs bond-then-cut with precise positioning, and together with the developed bridge-supporting algorithm about $30-80 \%$ waste material according to various geometric shapes can be removed online. Hollow pieces that are hardly produced by LOM process can also be easily produced by the new process. Hence, the proposed process promotes the performance efficiency of laminated process, the manufacturing ability regardless the geometry of the parts, and of material used. In the following sections, the new process is described, but the bridge-building algorithm is not presented for the conciseness of the paper.

\section{Online de-cubing process}

The mechanism of the proposed process is shown in Fig. 1. A self-adhesive sheet with shielding paper, shown in Fig. 2, is used as the working material, controlled by a feeding and a collecting roller to make it progresses and maintains constant tension. Below the material lies a platform for laminating while there is a plate, moving up and down to perform pressing, a laser cutter, moving in the $X-Y$ direction, and an inkjet above the material. Referring to Fig. 1, the com-

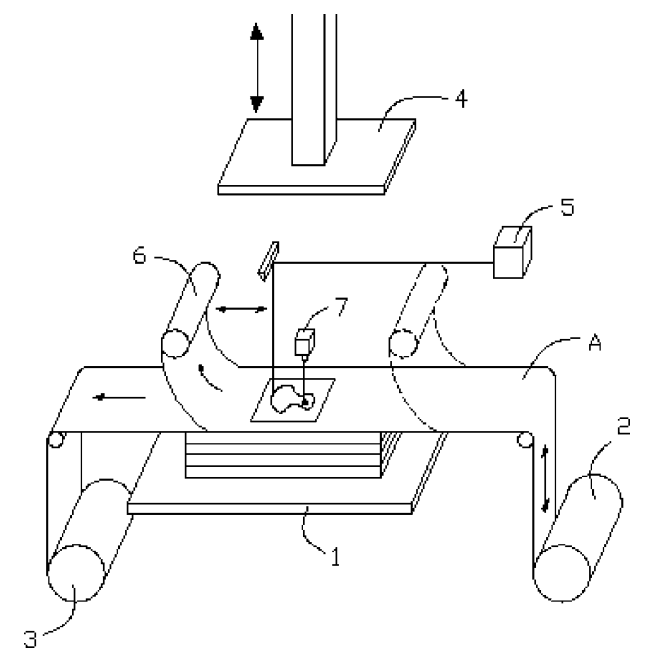

Fig. 1. The mechanism of the proposed process.

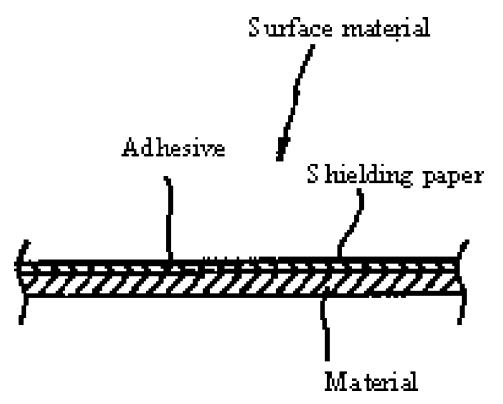

Fig. 2. Material structure.

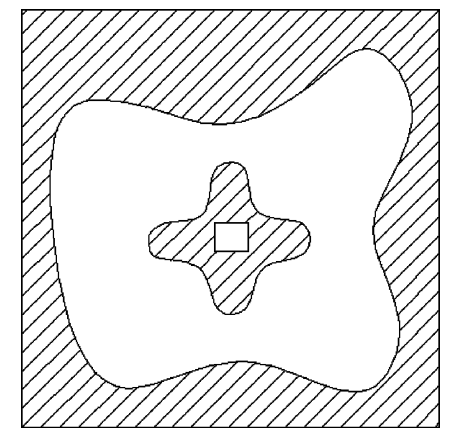

Fig. 3. The original drawing.

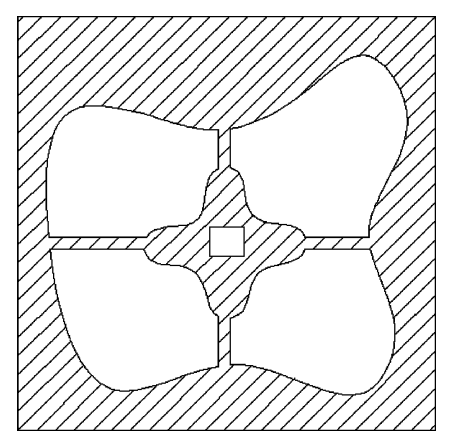

Fig. 4. The altered drawing.

ponents include: 1 , platform; 2 , feeding roller; 3 , collecting roller; 4 , plate; 5 , laser cutter; 6 , de-cubing device; 7 , inkjet.

The steps are detailed as follows.

(1) The 3D drawing of the workpiece is sliced into $2 \mathrm{D}$ contour drawings as shown in Fig. 3, in which the blank part represents the waste material. By means of the proposed algorithm, each 2D drawing is altered into drawing with or without bridges to help supporting and positioning the islands, shown in Fig. 4, and is output to the cutting unit. The aforementioned bridges will be removed after layers are laminated completely.

(2) After the material is positioned, a plate is employed to make it bond tightly with the previous layer. Then, the shielding paper is torn opposite to the feed direction as shown in Figs. 5 and 6.

(3) The 2D drawing in (1) is input to the laser cutter, being cut as required contour, as shown in Fig. 7.

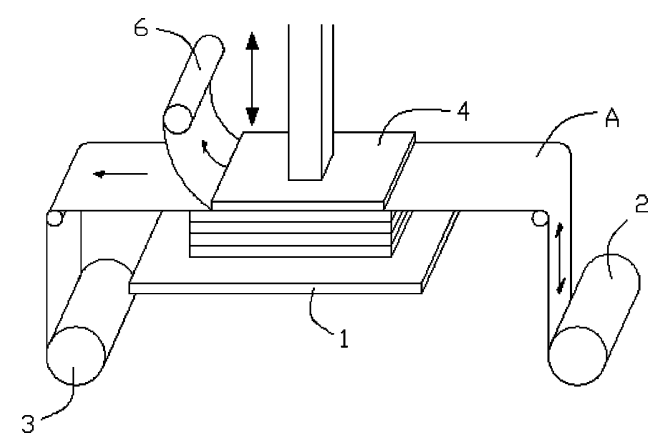

Fig. 5. Bonding. 


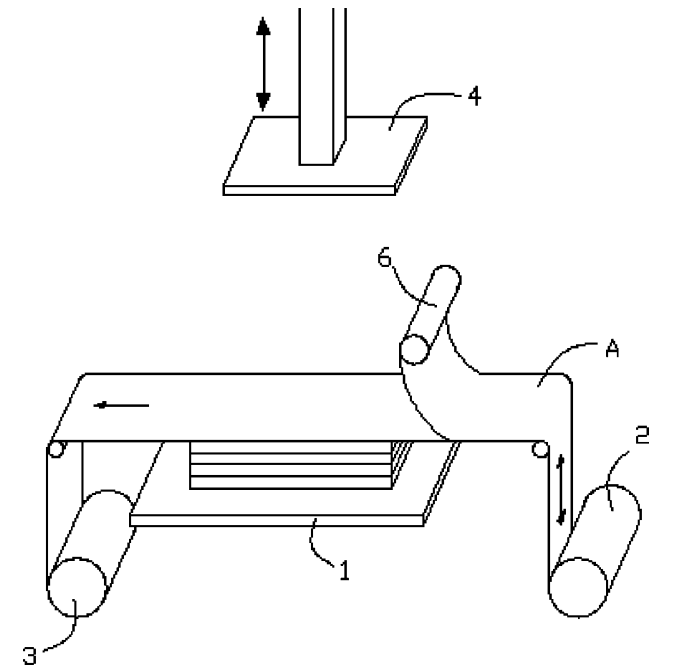

Fig. 6. The shielding paper is torn opposite to the feed direction.

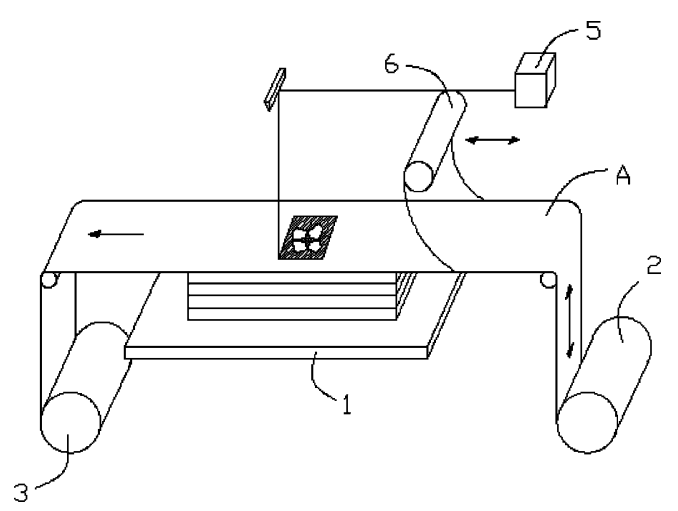

Fig. 7. Laser cutting.

(4) Carbon powders are jetted to part of the waste material region, which is needed to be "non-adhesive", reducing its adhesion to help de-cubing. As shown in Fig. 8, waste material $(i-j)$ in the 3rd layer has to be removed online while part of it may be bonded with the 2nd layer. Thus, region $i-j$ in the 2 nd layer has to be jetted with carbon powders to achieve non-adhesive, helping the 3rd layer to de-cube waste material. However, non-adhesive is not necessary for every layer to practice.

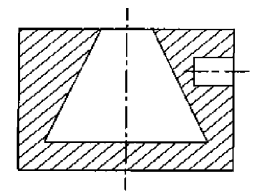

(a)

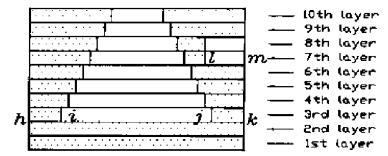

(b)

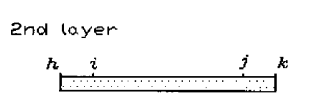

$i j$ t the burn out region

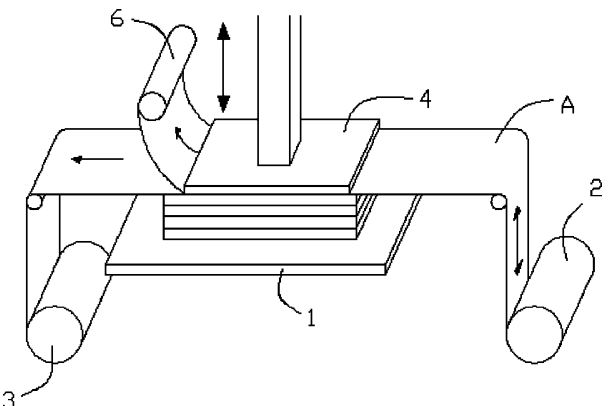

Fig. 9. The shielding paper is sent back by the roller and pressed by the plate again.

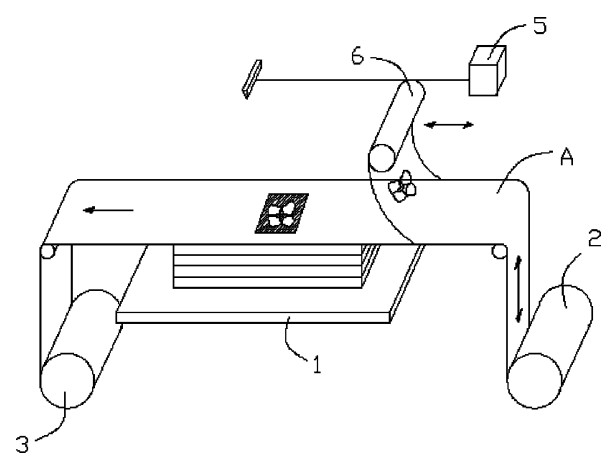

Fig. 10. Waste material is removed online.

(5) The shielding paper torn in (2) is sent back in the feed direction by the roller, and is pressed by the plate again, as shown in Fig. 9. Because the adhesion force between shielding paper and waste material is greater than that between waste material and the workpiece (non-adhesive has been practiced between them), the useless material is able to be removed online by tearing the shielding paper once again, as shown in Fig. 10.

(6) Steps (1)-(5) are performed repeatedly until the cutting and de-cubing are finished. Finally, a part is completed after little remained waste material and supporting bridges are removed.

There is one thing need to be taken notice of in the proposed process. As shown in Fig. 11, after the waste material in the 3rd layer is de-cubed, the waste material in the 4th layer should not be cut off completely in case it drops because of the weight.

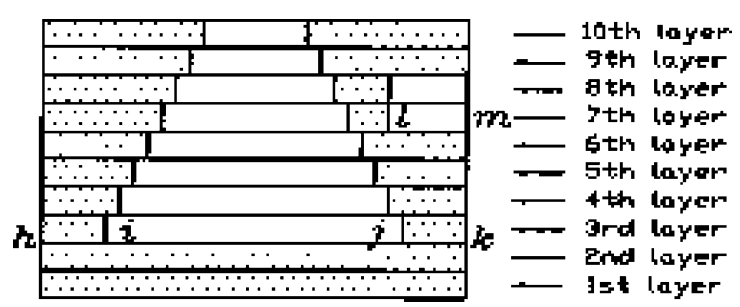

Fig. 11. The 4th-10th layer should not be cut off completely.

Fig. 8. Non-adhesive. 


\section{Experiments and results}

An experimental simulation is conducted to prove the feasibility of the proposed process, and the differences between LOM and the new process are discussed on the basis of the experimental results.

A calabash-shaped model and a ball-within hollow cuboid, as shown in Fig. 12(a) and (b), are chosen as parts to be manufactured in the experiment. The $0.092 \mathrm{~mm}$ thickness self-adhesive sheets with shielding paper (CLA5) are used as the working material for the proposed process, while $0.1 \mathrm{~mm}$ thickness LPH 042 14-LOM thermoplastic papers are employed as the working material of LOM.

First, the two models are produced by LOM, as shown in Fig. 13. Subsequently, in the proposed process, a bladed platform cutter is employed as the cutting unit. The procedures are as follows.

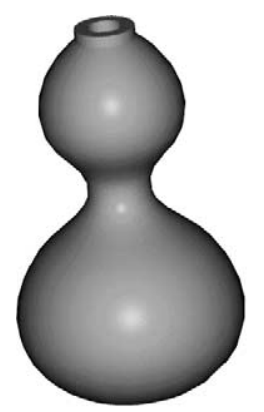

(a)

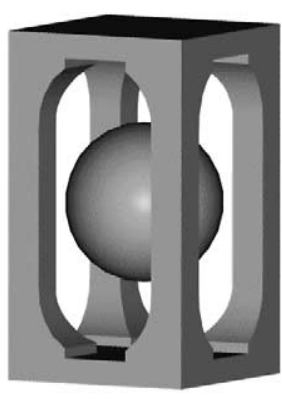

(b)
Fig. 12. 3D models: (a) a calabash-shaped model, (b) a ball-within hollow cuboid model.

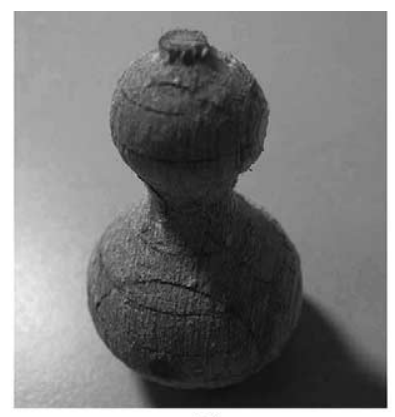

(a)

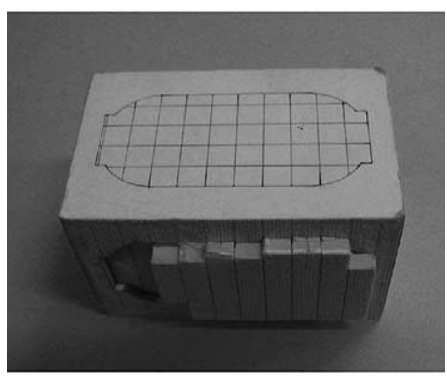

(b)

Fig. 13. Part produced by LOM: (a) a calabash-shaped workpiece, (b) a ball-within hollow cuboid workpiece.

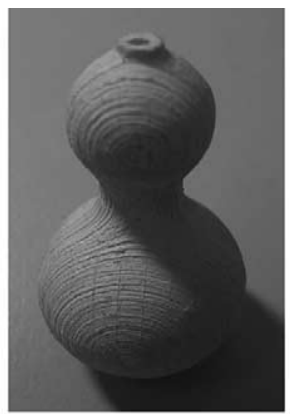

(a)

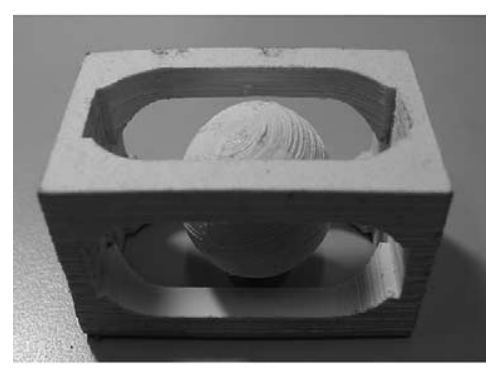

(b)

Fig. 14. Parts produced by the proposed process: (a) a calabash-shaped workpiece, (b) a ball-within hollow cuboid workpiece.

a. Drawings of the two models are completed with AutoCAD according to the proposed algorithm.

b. Positioning and bonding are performed at the same position of the platform.

c. Knife cutting is used to simulate the laser cutting.

d. The non-adhesive is practiced with a laser printer.

e. Steps of positioning, bonding, and cutting are carried out repeatedly until all layers are laminated completely.

f. Parts are produced as shown in Fig. 14 after all the supporting and positioning bridges are removed.

Comparing Figs. 13 and 14, it is obvious that parts produced by LOM have the problem that inner waste material is unable to be de-cubed; however, the problem does not exist in parts produced by the proposed process.

\section{Discussion}

According to the results from the experiment together with the proposed algorithm, characteristics, advantages and disadvantages of the two processes are compared as follows.

Table 1 shows the amount of waste material removed (WMR) and de-cubing time of the two processes. The proposed process not only shortens the time for post-processing, but also can remove $30-80 \%$ waste material online according to various geometric shapes.

Processing time is the sum of the time spent in preprocessing, cutting, bonding, and post-processing. The preprocessing for the proposed process is more complicated because of building bridges from the 2D drawings. Nonetheless, the computation time for the developed algorithm is 
Table 1

Comparison of de-cubing of the two processes

\begin{tabular}{llllll}
\hline & \multicolumn{2}{l}{ Amount of online WMR } & & \multicolumn{2}{l}{ Time for de-cubing } \\
\cline { 2 - 3 } & LOM & Proposed process (\%) & & LOM (min) & Proposed process (min) \\
\hline Calabash-shaped workpiece & NA & 50 & & 80 & 15 \\
Ball-within hollow cuboid workpiece & NA & 80 & Unable & 10 \\
\hline
\end{tabular}

Table 2

Overall comparison of the two processes

\begin{tabular}{llll}
\hline & & LOM & Proposed process \\
\hline Characteristics of material & $\begin{array}{l}\text { Position of adhesive } \\
\text { Type of adhesive } \\
\text { Rebonding due to rising } \\
\text { temperature after cutting }\end{array}$ & $\begin{array}{l}\text { Downward } \\
\text { Thermoplastic adhesive } \\
\text { Will occur }\end{array}$ & $\begin{array}{l}\text { Upward } \\
\text { Self-adhesive } \\
\text { Will not occur }\end{array}$ \\
& $\begin{array}{l}\text { Type of positioning } \\
\text { Heating device }\end{array}$ & Bond-then-cut & Hot roller \\
& $\begin{array}{l}\text { Online de-cubing } \\
\text { Laser power }\end{array}$ & No & Bond-then-cut \\
& Time for post-processing & Consuming & Long and difficult \\
& Manufacturing ability of & Hollow parts are hard to be produced & Yes \\
& geometric parts & Less consuming & Short and easy \\
& Hollow parts & Hollow parts can be produced easily \\
Characteristics of parts & Inner waste material cannot be removed & Inner waste material can be removed online \\
& Strength & Damage the part easily & Seldom damage the part \\
\end{tabular}

very limited due to the advent of high-speed CPU computer. Hence, the processing time of the two processes is about the same.

Both the two processes have to conduct crosshatching on the outer waste material. However, it is also necessary for LOM to practice crosshatching on the inner waste material while bridge cutting is the only thing left for the proposed process. Hence, $20-50 \%$ cutting time can be saved according to the geometric shapes by the use of the proposed process. As for the bonding time, there is no difference between the two processes. Accordingly, the proposed process has a great advantage in processing time.

In addition, most inner waste material is removed during the proposed process so that various geometric parts, such as hollow and shell-shaped shapes, can easily be produced.

From experiment and above-mentioned discussion, the characteristics of material, process, and parts of the proposed process are much different from LOM. The detailed comparison of the two processes is listed as Table 2.

\section{Conclusions}

On the basis of the proposed process with the bridge-supporting algorithm, about $30-80 \%$ waste material according to various geometric shapes can be removed during the process, the time and difficulty of post-processing are reduced greatly, and the efficiency is promoted.
Since inner waste material is de-cubed during the proposed process, the manufacturing ability regardless the geometry of the parts is improved efficiently. Thus, hollow and shell-shaped parts can be produced much easier. Besides, it is not necessary to perform crosshatching on all waste material during cutting; therefore, laser power and cutting time can be reduced effectively.

\section{References}

[1] I. Cho, K. Lee, Development of a new sheet deposition type rapid prototyping system, Int. J. Mach. Tool Manuf. 40 (12) (2000) 18131829.

[2] J. Hur, K. Lee, Efficient algorithm for automatic support structure generation in layered manufacturing, in: Proceedings of the 1996 ASME Design Engineering Technical Conference and Computers in Engineering Conference, Irvine, USA, 1996.

[3] Y.Y. Chiu, Y.S. Liao, C.C. Hou, The approach of on line waste removal process for bridged laminated object manufacturing (LOM), in: Proceedings of the 13th Solid Freedom Fabrication Symposium, University of Texas at Austin, USA, 5-7 August 2002.

[4] D. Klosterman, R. Chartoff, N. Osborne, G. Graves, A. Lightman, G. Han, Laminated object manufacturing of advanced ceramics and composites, in: Proceedings of the Seventh International Conference on Rapid Prototyping, Dayton, USA, 1997, pp. 43-50.

[5] C. Chi, L. Dodin, S. Pak, Development and fabrication of metallic LOM objects, in: Proceedings of the Seventh International Conference on Rapid Prototyping, Dayton, USA, 1997, pp. 293-299.

[6] Metal tape for LOM, Rapid Prototyping Report, Long Peak Engineering Inc., February, 1996. 